\section{PDE-5-Hemmer wirken auch ohne sexuelle Stimulation}

Für gewöhnlich wirken Viagra ${ }^{\circledR}$ \& Co. nur, wenn man sexuell stimuliert wird. In einem Laborsetting konnte nun gezeigt werden, dass mit PDE-5-Hemmern auch ohne sexuelle Stimulation eine rigide Erektion erreicht werden kann. Bei dauerhafter Gabe könnte sich so die Erektionsfähigkeit bessern, vermuten Forscher.

$\mathrm{M}$ it den Inhibitoren der Phosphodiesterase 5 (PDE-5), Sildenafil $\left(\right.$ Viagra $\left.^{\circledR}\right)$, Tadalafil $\left(\right.$ Cialis $\left.^{\circ}\right)$ und Vardenafil $\left(\right.$ Levitra $\left.{ }^{\star}\right)$, steht eine wirksame orale Behandlungsoption für die erektile Dysfunktion (ED) zur Verfügung. Studien haben ergeben, dass eine kontinuierliche Gabe von PDE-5-Hemmern einer Bedarfsmedikation überlegen sein könnte. So treten unter eine Langzeitgabe unter anderem häufigere nächtliche Spontanerektionen auf, die sich wiederum positiv auf die Funktion des Corpus cavernosum auswirken. Es wurde postuliert, dass sich durch das Ausbleiben von regelmäßigen sexuell provozierten oder nächtlichen Erektionen die Sauerstoffversorgung im Penis verschlechtert und die glatte Muskulatur abnimmt. Dadurch kann sich eine ED verschlechtern.

In einer aktuellen Studie wurden nun die Effekte von PDE-5-Hemmern auf verschiedene Parameter wie Erektionsdauer, Zeit bis zur ersten Erektion und Gesamtrigidität untersucht - und zwar ohne sexuelle Stimulation.

In die doppelblinde Studie wurden 80 Patienten eingeschlossen, die keine ED hatten, aber unter Ejaculatio praecox (< 1 min bis zur Ejakulation bei mehr als $50 \%$ der Geschlechtsverkehr-Versuche) litten. Diese Patienten stammten aus einer anderen Studie, in der der Einfluss von PDE-5-Inhibitoren auf Ejaculatio praecox untersucht wurde. Die Männer wurden in vier Gruppen eingeteilt und erhielten nach drei Tagen sexueller Enthaltsamkeit entweder Placebo, $50 \mathrm{mg}$ Sildenafil, $20 \mathrm{mg}$
Tadalafil oder $10 \mathrm{mg}$ Vardenafil. Im unmittelbaren Anschluss wurden die Probanden in einen stillen Raum gesetzt und aufgefordert, über einen Zeitraum von 1,5 Stunden Zeitschriften zu lesen, die keinerlei sexuell stimulierendes Material enthielten. Mit elastischen Messschlingen wurde die Rigidität der Erektion an Penisspitze und -basis gemessen (Rigiscan Plus ${ }^{\circledast}$.

$40 \%$ aus der Placebogruppe bekamen eine rigide Erektion, $71 \%$ aus der Sildenafil-Gruppe, $47 \%$ aus der Tadalafil-Gruppe und $70 \%$ aus der Vardenafilgruppe (p $=0,126)$. Bis die erste rigide Erektion der Penisbasis gemessen wurde, dauerte es

durchschnittlich 58,0 min (Placebo), 21,5 min (Sildenafil), 54,5 min (Tadalafil) beziehungsweise 57,0 min (Vardenafil) $(\mathrm{p}=$ 0,032 ). Die mittlere Erektionsdauer betrug 4,0 min unter Placebo, 27,5 min unter Sildenafil, 10,0 min unter Tadalafil und 11,5 min unter Vardenafil $(p=0,013)$. Die Forscher weisen darauf hin, dass der Messzeitraum von 90 Minuten für Tadalafil womöglich nicht ausreicht, um seine volle Plasmakonzentration zu erreichen.

Fazit: Mit allen drei Substanzen konnten binnen kurzer Zeit rigide Erektionen ohne sexuelle Stimulation erreicht werden, was den Einsatz der PDE-5-Hemmer bekräftigt. Jedoch sind noch weitere Studien nötig, um zu bestätigen, dass eine Langzeitgabe von PDE-5-Inhibitoren zur Behandlung einer ED nützlich ist.

Gökçe A et al. The effects of phosphodiesterase type 5 inhibitors on penile rigidity variables during a period with no sexual stimulation. BJUI 2011; 107: 264-7

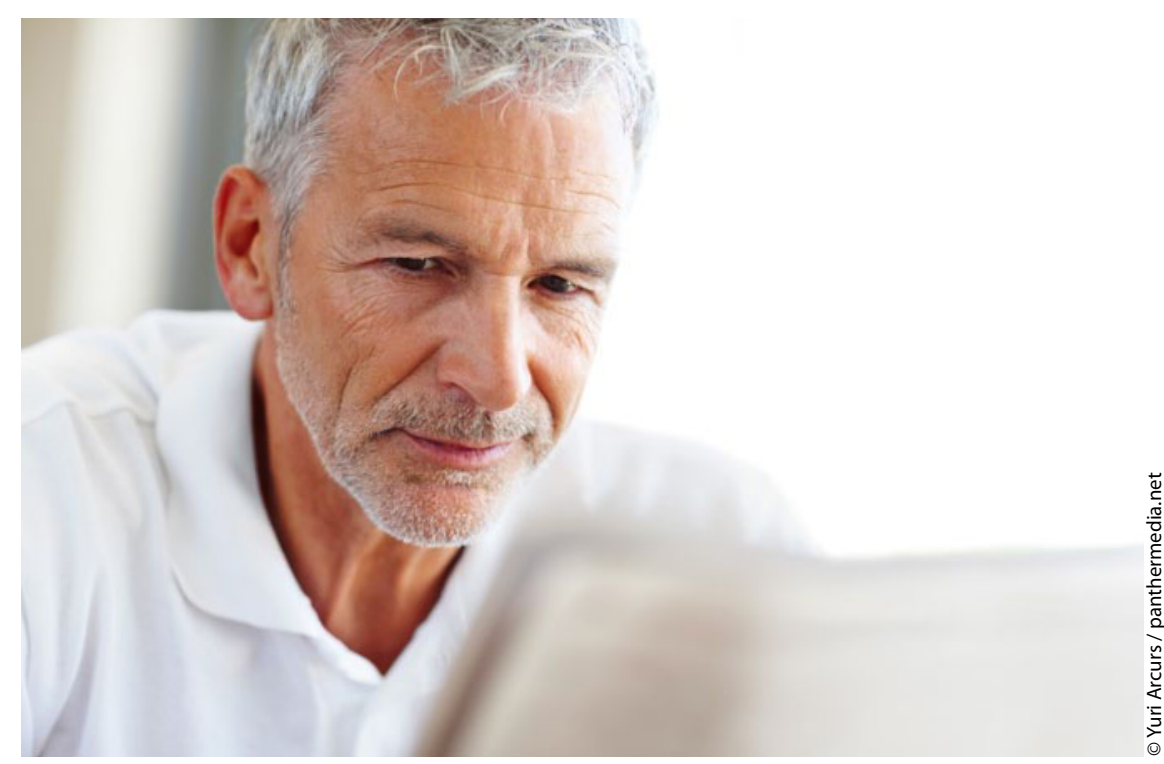

Mag die Lektüre noch so langweilig sein - einer aktuellen Studie zufolge bewirken PDE-5-Hemmer auch ohne sexuelle Stimulation eine Erektion. 